\title{
ORIGINAL
}

\section{COMPARACIÓN DE LOS MODELOS SCORE Y FRAMINGHAN EN EL CÁLCULO DE ALTO RIESGO CARDIOVASCULAR PARA UNA MUESTRA DE VARONES DE 45 Y 65 AÑOS DE ASTURIAS}

\author{
Artemio Álvarez Cosmea (1), Luis Díaz González (1), Vicente López Fernández (1), Miguel Angel \\ Prieto Díaz (2) y Saúl Suárez García (1) \\ (1) Centro de Salud Ventanielles-Colloto. Oviedo. Principado de Asturias. \\ (2) Centro de Salud Vallobín-Concinos. Oviedo. Principado de Asturias.
}

\section{RESUMEN}

Fundamento: Recientemente el Comité Español Interdisciplinario para la Prevención Cardiovascular (CEIP) elaboró un consenso para adaptar las recomendaciones de la Guía Europea de Prevención cardiovascular a la población española, utilizando el SCORE como método de cálculo de riesgo. El objetivo de este trabajo es conocer las diferencias que supone pasar del criterio de Framingham al de SCORE en la clasificación de los varones en el grupo de alto riesgo.

Métodos: Estudio descriptivo transversal realizado en atención primaria. El número de participantes fue de 379 varones de entre 45 y 65 años, correspondientes a 4 cupos de medicina general de 3 centros de salud del Principado de Asturias. Medidas principales: cálculo del riesgo cardiovascular y prevalencia de alto riesgo según Framingham clásico y según SCORE. Estimación de curva de prevalencia de alto riesgo por regresión logística.

Resultados: La prevalencia de alto riesgo según la ecuación de Framingham fue de $24 \%$ ( $\left.\mathrm{IC}_{95 \%}=19,9-28,7\right)$ y según SCORE de $17,9 \%\left(\mathrm{IC}_{95 \%}=14,3-22,3\right)$ siendo la diferencia estadísticamente significativa $(\mathrm{p}=0,02)$. El modelo SCORE presenta prevalencias menores en sujetos menores de 60 años y mayores por encima de los 60; en varones fumadores SCORE presenta prevalencias menores en personas menores de 58 años; los varones de alto riesgo con Framingham y bajo riesgo con SCORE son más jóvenes, fumadores y con cifras medias más bajas de presión arterial y cifras moderadas de colesterol.

Conclusiones: Al aplicar el modelo SCORE clasificamos menos varones como de alto riesgo, sobre todo en menores de 58 años, fumadores y con cifras moderadas de colesterol, que con la escala de Framingham.

Palabras clave: Factores de riesgo. Enfermedades cardiovasculares. Atención primaria de salud.

Correspondencia:

Artemio Álvarez Cosmea

C/ Rafael Altamira $5,11^{\circ} \mathrm{A}$

33006 Oviedo. Asturias

Teléfono: 985-253555

Correo electrónico: aalvarezco@papps.org
ABSTRACT

\section{Comparison of the SCORE and} Framingham Models in Calculating High Cardiovascular Risk for a Sample of Males within the 45-65 Age Range in Asturias, Spain

Background: The Spanish Interdisciplinary Committee for Cardiovascular Prevention has recently drafted a consensus for adapting the European Cardiovascular Prevention Guide to the Spanish population, using SCORE as the risk-calculation method. This study is aimed as ascertaining the differences involved in changing over from the Framingham to the SCORE criterion in the classification of males within the high-risk group.

Methods: Descriptive cross-sectional study conducted in primary care. A total of 379 males within the 45-65 age range, corresponding to four (4) groups from general practitioners from three healthcare centers in the Principality of Asturias. Main measurements: calculation of the cardiovascular risk and high-risk prevalence by the conventional Framingham method and according to SCORE. High-risk prevalence curve estimated by logic regression.

Results: The high-risk prevalence according to the Framingham equation was $24 \%($ CI95\% $=19.9-28.7$ ) and was $17.9 \%$ $(\mathrm{CI} 95 \%=14.3-22.3)$ for SCORE, the difference being statistically significant $(\mathrm{p}=0.02)$. The SCORE model shows lower prevalences among subjects under age 60 and higher for those over age 60 . Among male smokers, SCORE shows lower prevalences among individuals under 58 years of age, the high-risk males with Framingham and low-risk with SCORE being younger, smokers and having lower average blood pressure figures and moderate cholesterol figures.

Conclusions: On applying the SCORE model, we classify fewer males as high-risk, especially among those under 58 years of age who are smokers having moderate cholesterol figures than when the Framingham scale is used.

Key words: Risk factors. Cardiovascular diseases. Primary health care. 


\section{INTRODUCCIÓN}

Desde la publicación de la guía de prevención cardiovascular de las Sociedades Europeas (Third Task Force European, 2003) ${ }^{1}$ se recomienda la utilización del $\mathrm{SCORE}^{2}$ para el cálculo de riesgo cardiovascular, aunque en realidad lo que mide es mortalidad cardiovascular. Hasta ahora se utilizaban las tablas de riesgo de Framingham ${ }^{3}$ para la detección de pacientes de alto riesgo, ya que eran las recomendadas por el Programa de Actividades Preventivas y Promoción de la Salud (PAPPS) ${ }^{4}$ de la Sociedad Española de Medicina de Familia y más recientemente por el Programa Integral de la Cardiopatía Isquémica (PICI) ${ }^{5}$ del Ministerio de Sanidad , aunque sabíamos por diferentes estudios que, excepto en el Norte de Europa ${ }^{6}$, sobreestiman el riesgo en la mayoría de poblaciones europeas, sobre todo en las mediterráneas ${ }^{7-10}$. El Comité Español Interdisciplinario para la Prevención Cardiovascular (CEIPC) ha consensuado la adaptación de la guía de prevención cardiovascular europea a la población española, recomendando el método SCORE para el cálculo de riesgo cardiovascular (tablas para países de bajo riesgo ${ }^{11}$. Recientemente se han publicado estudios ${ }^{12}$ que valoran el cambio del método de Framingham al SCORE. En la práctica clínica supone tratar un número menor de sujetos varones de mediana edad con cifras de colesterol total altas.

Dado que no podemos comparar el riesgo calculado con uno y otro método, ya que miden cosas distintas (Framingham mide el riesgo coronario total y SCORE la mortalidad cardiovascular), nos propusimos como objetivo conocer las implicaciones en la clasificación de los varones como de alto riesgo, dato fundamental a la hora de instaurar tratamiento con fármacos de los distintos factores de riesgo (FR), sobre todo la hipertensión arterial y la hipercolesterolemia. Además, precisamente es en la población masculina en la que existen evidencias claras de que en prevención primaria las estati- nas disminuyen la morbimortalidad cardio$\operatorname{vascular}^{13,14}$.

\section{SUJETOS Y MÉTODOS}

Se realizó un estudio transversal en el ámbito de la atención primaria, siendo la población de estudio todos los varones entre 45 y 65 años de edad de cuatro cupos de medicina general atendidos en tres centros de salud de la Comunidad Autónoma del Principado de Asturias. Para confeccionar el listado de sujetos utilizamos la base de datos de la tarjeta sanitaria de los referidos cupos, habiendo en total 600 varones. Consideramos criterios de exclusión: ser enfermo terminal, estar inmovilizado, ser alcohólicos, tener trastornos mentales graves y no residir en la zona. Los sujetos se seleccionaron a partir de la consulta a demanda, y a los que no acudían se les citaba por teléfono a consulta para realizar el estudio completo. El período de estudio fue de enero a diciembre de 1999.Se recogieron las siguientes variables: edad, antecedentes familiares en primer grado de enfermedad coronaria precoz o muerte súbita en varones menores de 55 años y en mujeres menores de 65 años, antecedentes personales documentados de patología cardiovascular (cardiopatía isquémica, accidente cerebrovascular, insuficiencia cardiaca e isquemia crónica de miembros inferiores), medida de presión arterial sistólica y diastólica (se descartaba la primera toma en consulta y se realizaron otras dos tomas, siendo el resultado final la media de estas dos últimas), diabetes (se consideró como diabéticos a aquellas personas ya diagnosticadas o con tratamiento antidiabético; para el nuevo diagnóstico se utilizaron los criterios de la OMS), hábito tabáquico (se consideró fumadores a aquéllos que habían fumado al menos 1 cigarrillo en el último mes) y perfil lipídico completo (colesterol total, triglicéridos, HDL-colesterol, LDL-colesterol).

Para el cálculo de riesgo cardiovascular se utilizaron 2 escalas de riesgo: 1) método de 
Framingham clásico (Anderson, 1991) ${ }^{3}$ y 2) método SCORE ${ }^{2}$ : utilizamos la tabla para países de bajo riesgo, según el panel de colesterol total. Siguiendo las recomendaciones del CEIPC no se extrapolaron los resultados a la edad de 60 años. Para el cálculo del SCORE utilizamos el Heartscore de la página web de la Sociedad Europea de Cardiología ${ }^{15}$. La población diana fueron todos los varones sin enfermedad cardiovascular previa ni diabetes, ya que estos últimos también se consideran pacientes de alto riesgo cardiovascular y por lo tanto no se les puede aplicar el SCORE ${ }^{1}$.

Se clasificó a los sujetos como de alto riesgo si presentaban un riesgo mayor o igual al 20\% con el método de Framingham clásico ${ }^{3}$ o un riesgo de mortalidad cardiovascular mayor o igual al 5\% con SCO$\mathrm{RE}^{2}$.

Análisis estadístico: se calculan la media y desviación estándar de las variables cuantitativas y los porcentajes de la cualitativas. Para el cálculo de los intervalos de confianza (IC) de una proporción se utilizó la aproximación cuadrática de Fleiss ${ }^{16}$. Para comparar medias (dos categorías) se utilizó el test de la t de Student y para más de dos categorías se utilizó el test de Anova. Para valorar la asociación de variables cualitativas se utilizó la $\mathrm{chi}^{2}$ (para muestras independientes). Para comparar los resultados de alto riesgo por el método de Framingham clásico y por SCORE se utilizó el test de Mc Nemar (para muestras apareadas). La estimación de la curva de prevalencia de alto riesgo se realizó mediante regresión logística, siendo la variable dicotómica dependiente (alto riesgo $=1$, si el sujeto tenía alto riesgo de presentar algún evento cardiovascular en los siguientes años; alto riesgo $=0$, en caso contrario) y la independiente la edad del paciente ${ }^{17}$. La curva de prevalencia de alto riesgo viene dada por la expresión logística:

$$
\mathrm{P}(\mathrm{Y}=1)=1 / 1+\exp (-\alpha-\beta \mathrm{X})
$$

Donde $\mathrm{Y}$ es el estatus respecto al alto riesgo $(1=$ sí; $0=$ no) en el momento del estudio, y X la edad del paciente en años, y $\alpha$ y $\beta$ los coeficientes de regresión logística. Calculamos esta expresión para el alto riesgo diagnosticado por el método de Framingham clásico y por el SCORE. Una vez obtenidas las estimaciones de los coeficientes de la regresión logística $\alpha$ y $\beta$, y dando valores a la variable X (edad) se obtiene la prevalencia de alto riesgo para cada edad. Para valorar la fiabilidad entre ambos métodos se utilizó el porcentaje de concordancia global, el de cada grupo y el coeficiente Kappa. El análisis de los datos se realizó con los programas estadísticos SPSS (versión 11.0) y EPI INFO 6 (versión 6.04b).

\section{RESULTADOS}

De los 600 varones iniciales 44 no aceptaron participar, 34 no se captaron, 18 vivían fuera de zona, 10 tenían neoplasias de mal pronóstico, 5 por consumo excesivo de alcohol y 2 habían fallecido (se perdieron 113 (19\%) participantes en total). De los 487 restantes fueron excluidos $48(9,8 \%)$ por patología cardiovascular manifiesta; 47 (9,6\%) por ser diabéticos sin enfermedad cardiovascular (el SCORE los considera de alto riesgo) y 13 casos por faltar alguna de las variables, por lo que la muestra final estaba formada 379 participantes. En la tabla 1 se recogen sus características generales. La prevalencia de alto riesgo según la ecuación de Framingham fue de $24,0 \%$ (IC del $95 \%=19,9-28,7)$ y según SCORE de $17,9 \%$ (IC del 95\%=14,3-22,3) con diferencia estadísticamente significativa $(\mathrm{p}=0,02)$. En la figura 1 se recogen las curvas de prevalencia estimadas por regresión logística según la edad y los dos métodos diagnósticos de alto riesgo. En la tabla 2 se representa la concordancia de sujetos de alto y bajo riesgo según ambos métodos. El coeficiente Kappa de concordancia fue de 26,4. En la tabla 3 se muestra la prevalencia estimada con su intervalo de confianza para edades concretas 
Tabla 1

Características de los sujetos de la muestra

\begin{tabular}{|l|c|}
\hline \multicolumn{1}{|c|}{ Características } & $\begin{array}{c}\text { Hombres } \\
\text { n (\%) }\end{array}$ \\
\hline Edad media (DE) años & $54,2(6,6)$ \\
PAS media (DE) mmHg & $128,4(15,8)$ \\
PAD media (DE) mmHg & $78,0(9,4)$ \\
Colesterol media (DE) mg/dl & $230,4(39,7)$ \\
Riesgo Framingham, media (DE) & $14,9(8,9)$ \\
Riesgo SCORE, media (DE) & $2,9(2,6)$ \\
Fumadores n (\%) & $180(47,5)$ \\
Hipertensión arterial n (\%) & $85(22,4)$ \\
Obesidad (\%) & $109(29,0)$ \\
Colesterol $\geq 200 \mathrm{mg} / \mathrm{dl} \mathrm{n}(\%)$ & $294(77,6)$ \\
Colesterol $>250 \mathrm{mg} / \mathrm{dl} \mathrm{n}(\%)$ & $106(28,0)$ \\
\hline
\end{tabular}

DE: desviación estándar; PAS: presión arterial sistólica; PAD: presión arterial diastólica; Hipertensión arterial: PAS $\geq 140$ $\mathrm{mmHg}$ y/ o PAD $\geq 90 \mathrm{mmHg}$ ó tratamiento antihipertensivo; Obesidad: índice de masa corporal (IMC) $\geq 30 \mathrm{Kg} / \mathrm{Talla} \mathrm{m}^{2}$.

Tabla 2

Tabla de concordancia de pacientes con riesgo alto según la ecuación de Framingham y SCORE

\begin{tabular}{|c|c|c|c|}
\hline & Framingham alto & Framingham bajo & Total \\
\hline $\begin{array}{c}\text { SCORE alto } \\
(\% \text { del total })\end{array}$ & 33 & 35 & 68 \\
$(8,7 \%)$ & $(9,2 \%)$ & $(17,9 \%)$ \\
\hline $\begin{array}{c}\text { SCORE bajo } \\
(\% \text { del total })\end{array}$ & 58 & 253 & 311 \\
\hline Total & $(15,3 \%)$ & $(66,8 \%)$ & 379 \\
$(\%$ del total $)$ & 91 & 288 & \\
\hline
\end{tabular}

Kappa $=26,4$ (error estándar de Kappa $=0,05$ ); Riesgo alto de Framingham: riesgo $\geq 20 \%$ a los 10 años; Riesgo alto de SCORE: riesgo $\geq 5 \%$ a los 10 años.

por los dos métodos. En la tabla 4 se muestra la comparación entre los grupos discordantes de alto riesgo con sus intervalos de confianza. Los grupos 1 (alto riesgo en ambos métodos) y 3 (alto riesgo en SCORE y bajo riesgo en Framingham) presentan medias más elevadas de edad, de presión arterial sis- tólica, de colesterol y de diagnósticos de hipertensión arterial que el grupo 2 (alto riesgo en Framingham y bajo riesgo en SCORE). El grupo 3 (alto riesgo en SCORE y bajo riesgo en Framingham) presenta menor prevalencia de fumadores. En pacientes más jóvenes y en fumadores es más pre- 
Tabla 3

Estimación de la prevalencia de alto riesgo según SCORE y Framingham para distintas edades en años

\begin{tabular}{|c|c|c|}
\hline $\begin{array}{c}\text { Edad } \\
\text { (años) }\end{array}$ & $\begin{array}{c}\text { Prevalencia alto riesgo } \\
\text { Framingham (IC del 95\%) }\end{array}$ & $\begin{array}{c}\text { Prevalencia alto riesgo } \\
\text { SCORE (IC del 95\%) }\end{array}$ \\
\hline 45 & $16,7(11,7-23,3)$ & $0,2(0,03-0,7)$ \\
50 & $20,3(16,4-24,9)$ & $1,0(0,4-2,7)$ \\
55 & $24,3(21,4-27,8)$ & $6,0(3,4-10,5)$ \\
60 & $29,2(24,6-34,2)$ & $29,4(24,4-34,9)$ \\
65 & $34,3(26,4-43,2)$ & $72,9(63,9-80,5)$ \\
\hline
\end{tabular}

$\mathrm{IC}=$ intervalo de confianza

Tabla 4

Comparación entre los grupos discordantes de riesgo alto

\begin{tabular}{|c|c|c|c|}
\hline Características & $\begin{array}{c}\text { Grupo 1 } \\
\text { n = 33 } \\
(\text { IC } 95 \%)\end{array}$ & $\begin{array}{c}\text { Grupo } 2 \\
\mathbf{n}=\mathbf{5 8} \\
(\text { IC } 95 \%)\end{array}$ & $\begin{array}{c}\text { Grupo } 3 \\
\mathbf{n}=\mathbf{3 5} \\
(\text { IC } 95 \%) \\
\end{array}$ \\
\hline Edad media años & $62,1(61,1-63,1)^{*}$ & $52,2(50,7-53,7)^{*}$ & $62,7(61,8-63,6)^{*}$ \\
\hline PAS media mmHg & $137,9(133,8-42,0) *$ & $127,7(124,6-130,8)^{*}$ & $137,1(130,7-143,5)^{*}$ \\
\hline PAD media mmHg & $81,5(78,2-84,8)$ & $79,6(77.6-81,6)$ & $81,3(77,8-84,8)$ \\
\hline Colesterol media mg/dl & $254,9(242,9-266,9) *$ & $224,8(212,5-227,1)^{*}$ & $241,7(229,8-253,6)^{*}$ \\
\hline Fumadores $\%$ & $87,9(70,9-96,0)^{*}$ & $74,1(60,7-84,4)^{*}$ & $51,4(34,3-68,3)^{*}$ \\
\hline Hipertensión arterial \% & $45,5(28,5-63,4)$ & $27,6(17,0-41,1)$ & $37,1(22,0-55,1)$ \\
\hline Obesidad \% & $24,2(11,7-42,6)$ & $24,1(14,3-37,5)$ & $22,9(11,0-40,6)$ \\
\hline Colesterol $\geq 200 \mathrm{mg} / \mathrm{dl}$ & $97,0(82,5-99,8)^{*}$ & $62,1(48,3-74,2)^{*}$ & $91,4(75,8-97,8)^{*}$ \\
\hline Colesterol $>250 \mathrm{mg} / \mathrm{dl}$ & $57,6(39,4-74,0) *$ & $25,9(15,4-39,3)^{*}$ & $40,0(24,4-57,8)^{*}$ \\
\hline $\mathrm{RF}$, media & $29,2(26,9-31,5)^{*}$ & $27,1(25,5-28,7)^{*}$ & $14,6(13,2-16,0)^{*}$ \\
\hline RSCORE, media & $8,1(7,3-9,0)^{*}$ & $2,2(1,9-2,5)^{*}$ & $6,7(5,8-7,6)^{*}$ \\
\hline
\end{tabular}

-IC 95\%: intervalo de confianza del 95\%; RF: riesgo Framingham; RSCORE: riesgo SCORE; PAS: presión arterial sistólica; PAD: presión arterial diastólica; Hipertensión arterial: $\mathrm{PAS} \geq 140 \mathrm{mmHg}$ y/ o $\mathrm{PAD} \geq 90 \mathrm{mmHg}$.

-Grupo 1: ambos métodos coinciden en catalogar al paciente de riesgo

-Grupo 2: Framingham riesgo alto y SCORE riesgo bajo

-Grupo 3: Framingham riesgo bajo y SCORE riesgo alto

_*”p $<0,05$ ", diferencia estadísticamente significativa entre los grupos. Para comparar las medias se utilizó el test de ANOVA, en el caso de que las varianzas no fueran homogéneas o la variable no tuviera una distribución normal se ha utilizado el test de KruskalWallis. Para valorar la homogeneidad de las varianzas se utilizó el test de Bartlett's. Para la comparación de porcentajes se utilizó el test de la $\mathrm{X}^{2}$.

valente el alto riesgo por el método de la ecuación de Framingham que por el SCORE. En la figura 2 se presentan las curvas de prevalencia estimadas por regresión logística para ambos métodos de cálculo de alto riesgo en fumadores y no fumadores. Comparado con el método SCORE, el método Framingham presenta mayores prevalencias de alto riesgo (más del 20\%) en pacientes menores de 58 años y fumadores.

\section{DISCUSIÓN}

Entre las limitaciones del estudio figuran: no se puede aplicar a la población general, ya que el estudio se hizo sólo en varones de 45 a 65 años; no podemos comparar directamente el riesgo calculado con uno y otro método, ya que miden cosas distintas (Framingham, riesgo coronario total y SCORE, mortalidad cardiovascular); las curvas de 
Figura 1

Estimación de la curva de prevalencia de alto riesgo según SCORE y ecuación de Framingham según edad

Prevalencia $(\%)$

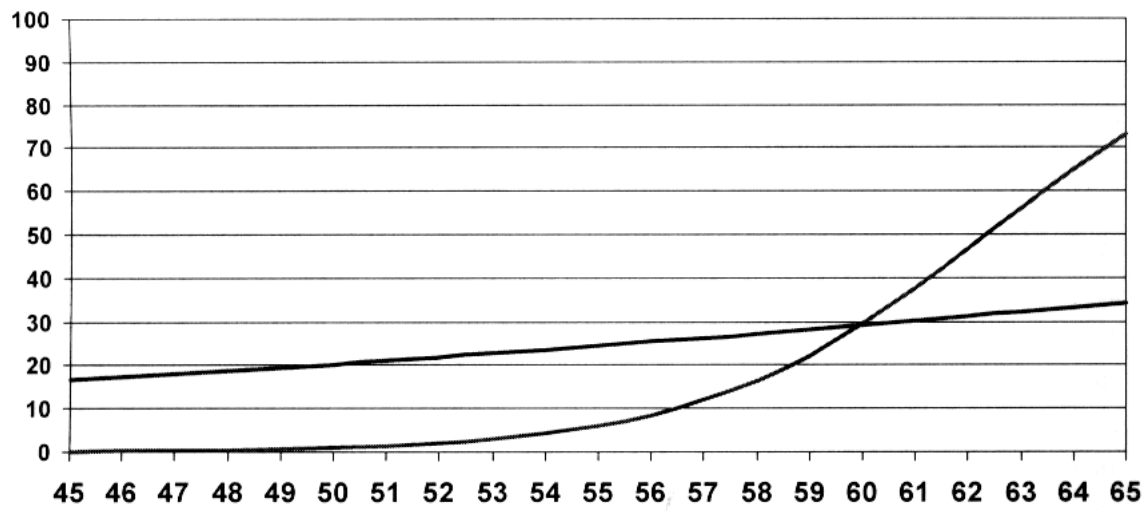

SCORE -Framingham

Años

* Estimación de la curva de prevalencia de alto riesgo de Framingham: $\mathrm{P}(\mathrm{Y}=1)=1 / 1+\mathrm{EXP}-($ constante $)-(0,048$ * $($ edad $))$; " $\mathrm{Y}=1$ " $=$ riesgo Framingham $\geq 20 \%$ a los 10 años; test de bondad de ajuste de Hosmer Lemeshow $=12,56(p=0,12)$

Variables en la ecuación

\begin{tabular}{|l|c|c|c|}
\hline & Beta & eeb & "p" \\
\hline Edad & 0,048 & 0,018 & 0,009 \\
\hline Constante & $-3,768$ & 1,014 & 0,000 \\
\hline
\end{tabular}

* Estimación de la curva de prevalencia de alto riesgo según ecuación de SCORE: $\mathrm{P}(\mathrm{Y}=1)=1 / 1+\mathrm{EXP}-(-23,316)-(0,374$ * (edad)); "Y=1" = riesgo SCORE $\geq 5 \%$ a los 10 años ; test de bondad de ajuste de Hosmer Lemeshow $=3,05(\mathrm{p}=0,93)$ Variables en la ecuación

\begin{tabular}{|l|c|c|c|}
\hline & Beta & eeb & “p” \\
\hline Edad & 0,374 & 0,047 & 0,000 \\
\hline Constante & $-23,316$ & 2,851 & 0,000 \\
\hline
\end{tabular}

$*$ eeb $=$ error estándar del coeficiente beta

$*$ Beta $=$ coeficiente beta

prevalencia son una estimación; el coeficiente Kappa presenta limitaciones para medir la concordancia ${ }^{18}$.

Aunque lo ideal es conocer el riesgo cardiovascular global de una persona, es difícil decidirse por un método u otro, y elegir un punto de corte de alto riesgo cardiovascular ${ }^{19-22}$. A pesar de esto conocemos por diversos estudios que la aplicación de la ecuación de Framingham en población europea sobreestima el riesgo cardiovascular, sobre todo en países mediterráneos de bajo riesgo como el nuestro; de ahí que parezca lógico que aunque midan distintos tipos de riesgo, el método Framingham clasifique a más sujetos como de alto riesgo (24\%) que el SCORE $(17,9 \%)$, ya que el SCORE ha sido elaborado a partir de cohortes de 12 países europeos. En la figura 1, que representa la estimación de las curvas de prevalencia de alto riesgo según ambos métodos, destaca que utilizando el SCORE la prevalencia de alto riesgo es menor en varones menores de 60 años, por lo que les vamos a dejar de tratar con fármacos al no considerarlos de alto 
Figura 2

Estimación de las curvas de prevalencia de alto riesgo según SCORE y Framingham en fumadores y en no fumadores

Prevalencia (\%)
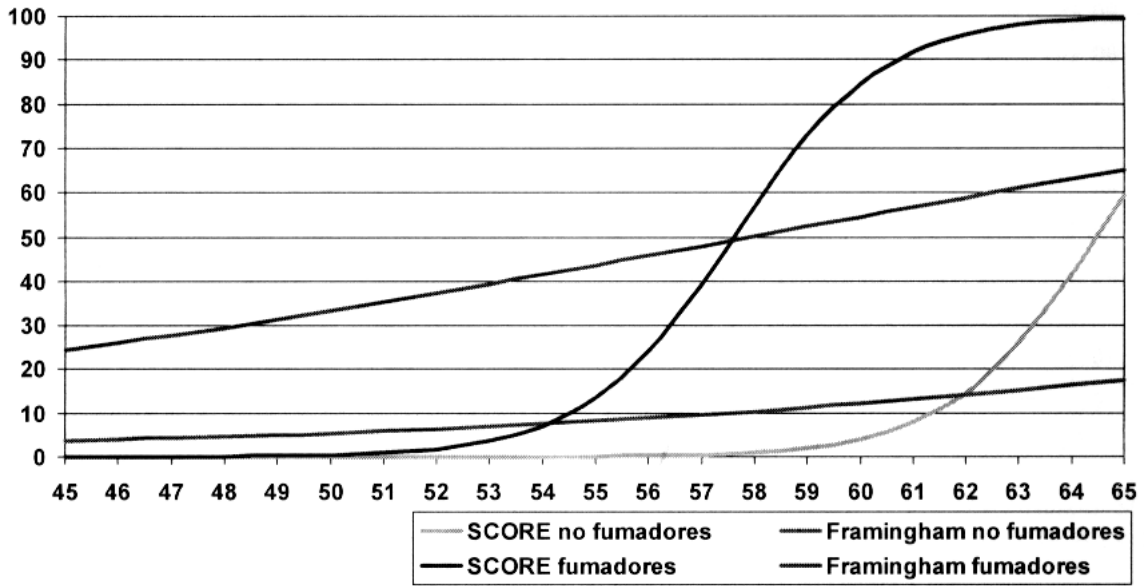

Años

* Estimación de la curva de prevalencia de alto riesgo SCORE: $\mathrm{P}(\mathrm{Y}=1)=1 / 1+\mathrm{EXP}-($ constante $)-(0,713 *$ (edad) $)-(4,893 *$ (tabaco)); "Y=1" = riesgo SCORE $\geq 5 \%$ a los 10 años; edad en años; en fumadores, tabaco $=1$; en no fumadores, tabaco $=0$; test de bondad de ajuste de Hosmer Lemeshow $=3,12(\mathrm{p}=0,92)$

Variables en la ecuación

\begin{tabular}{l|c|c|c|}
\hline & Beta & eeb & “p” \\
\hline Edad & 0,713 & 0,107 & 0,000 \\
\hline Tabaco & 4,893 & 0,829 & 0,000 \\
\hline Constante & $-45,968$ & 6,820 & 0,000 \\
\hline
\end{tabular}

* Estimación de la curva de prevalencia de alto riesgo según ecuación de Framingham: $\mathrm{P}(\mathrm{Y}=1)=1 / 1+\mathrm{EXP}$ - (constante) - $(0,088$ * $($ edad $))-(2,166 *$ (tabaco $)) ; " Y=1 "=$ riesgo Framingham $\geq 20 \%$ a los 10 años; edad en años; en fumadores, tabaco $=1$; en no fumadores, tabaco $=0$; test de bondad de ajuste de Hosmer Lemeshow $=5,23(p=0,92)$.

Variables en la ecuación

\begin{tabular}{l|c|c|c|}
\hline & Beta & eeb & "p" \\
\hline Edad & 0,088 & 0,021 & 0,000 \\
\hline Tabaco & 2,166 & 0,309 & 0,000 \\
\hline Constante & $-7,264$ & 1,258 & 0,000
\end{tabular}

*eeb $=$ error estándar del coeficiente beta

$*$ Beta $=$ coeficiente beta

riesgo cardiovascular; al considerar la variable fumador (si/no), representada en la figura 2, utilizando el método SCORE con respecto a Framingham, la diferencia de prevalencia de alto riesgo es aún mayor.

Esta población (varones entre 40 y 60 años, fumadores, con cifras medias de colesterol) se podría considerar representativa de la población diana de las actividades preventivas a realizar en la consulta diaria, se considerarían de menor riesgo utilizando el SCORE.

En general, las características de los sujetos que dejamos de clasificar como de alto riesgo (grupo 2, alto riesgo según Framingham y bajo riesgo según SCORE), van a ser: varones de 52 años de media de edad, con un colesterol de $224 \mathrm{mg} / \mathrm{dl}$, fumadores (74\%) y con cifras normales de presión arterial, 
características similares a los datos del estudio de Maiqués et $\mathrm{al}^{12}$.

Por otro lado, según vemos en la figura 1 , el SCORE a partir de los 60 años sobreestima el riesgo si lo comparamos con Framingham, por lo que vamos a clasificar y tratar a más pacientes de alto riesgo a partir de esta edad, datos que coinciden con el estudio de Mostaza et $\mathrm{al}^{23}$, donde concluyen que con el SCORE se triplica el número de varones de edad avanzada de alto riesgo, aunque en este caso, se compara con la función de Framingham del ATP III ${ }^{24}$.

Se debe realizar en el futuro un análisis riguroso de las consecuencias de la aplicación del método SCORE por diversos motivos: se basa en datos de mortalidad y establece alto riesgo si la probabilidad de morir por enfermedad cardiovascular es igual o mayor al 5\%, no teniendo en cuenta la morbilidad, dato que algunos autores consideran de gran importancia, ya que la morbilidad en España supone el $75 \%$ de la morbimortalidad cardiovascular, con una gran repercusión socioeconómica ${ }^{25}$. Presenta la limitación de la edad (40-65 años) para su aplicación en la práctica clínica, aunque se podría considerar que el riesgo en personas mayores de 65 años es como mínimo similar al de las personas de 65 años. En pacientes con dislipemia deja de clasificar como de alto riesgo a varones de mediana edad y fumadores, donde los ensayos clínicos han demostrado gran eficacia de los tratamientos.

Aunque hoy en día se ha llegado a un consenso para utilizar el SCORE, se debe valorar su aplicación en la práctica clínica y esperar al desarrollo de otros métodos de cálculo de riesgo realizados en nuestro país (tablas de riesgo adaptadas a la población española: REGICOR ${ }^{9}$ ó DORICA ${ }^{26}$ ).

\section{BIBLIOGRAFÍA}

1. Executive Summary. Third Joint Task Force of European and other Societies on Cardiovascular
Disease Prevention in Clinical Practice. European guidelines on cardiovascular disease prevention in clinical practice. Eur Heart J 2003; 24: 1601-10.

2. Conroy RM, Pyöräla K, Fitzgerald AP, Sans S, Menotti A, De Backer G et al. Estimation of tenyear risk of fatal cardiovascular disease in Europe: the SCORE project. Eur Heart J 2003; 24: 9871003.

3. Anderson KM, Wilson PWF, Odell PM, Kannel WB. An Updated coronary risk profile. A Statement for health profesionals. Circulation 1991; 83: 356-62.

4. Villar Álvarez F, Maiqués Galán A, Brotons Cuixart C, Torcal Laguna J, Ortega Sánchez-Pinilla R, Vilaseca Canals J, et al. Actividades preventivas cardiovasculares en atención primaria. Programa de Actividades Preventivas y Promoción de la Salud, actualización 2003. Aten Primaria 2003; 32 (suppl 2):15-29.

5. Plan Integral de Cardiopatía Isquémica 2004-2007. Madrid: Ministerio de Sanidad y Consumo; 2003.

6. Haq IU, Ramsay LE, Yeo WW, Jackson PR, Wallis EJ. Is the Framingham Risk Funtion valid for Norther European populations? A comparison of methods for estimating absolute coronary risk in high risk men. Heart 1999; 81: 40-6.

7. Menotti A, Pudd PE, Lanti M. Comparison of the Framingham risk function- based coronary chart with risk function from an Italian population study. Eur Heart J 2000; 21: 365-70.

8. Elosua R, Belmonte E, Sentí M, Masiá R y Marrugat J. ¿Debe diseñarse una estrategia específica de prevención primaria de la cardiopatía isquémica para los países mediterráneos de Europa? Clin. Invest Arterioscl 2000; 12: 43-55.

9. Marrugat J, Solanas P, D’Agostino R, Sullivan L, Ordovás J, Cordón F et al. Estimación del riesgo coronario en España mediante la ecuación de Framingham calibrada. Rev Esp Cardiol 2003; 56: 253-61.

10. Ramos R, Solanas P, Cordón F, Rolfs I, Elosua R, Sala $\mathrm{J}$ et al. Comparación de la función de Framingham original y la calibrada del REGICOR en la predicción del riesgo coronario poblacional. Med Clin (Barc) 2003; 121: 521-6.

11. Brotons C, Royo-Bordonada MA, Álvarez-Sala L, Armario P, Artigao RR, Conthe P et al. Adaptación española de la Guía Europea de Prevención Cardiovascular. Comité Español Interdisciplinario para la 
Prevención Cardiovascular (CEIPC). Aten Primaria 2004 ; 34: 427-32.

12. Maiques A, Antón F, Franch M, Albert X, Aleixandre E, Collado A. Riesgo cardiovascular del SCORE comparado con el de Framingham. Consecuencias del cambio propuesto por las Sociedades Europeas. Med Clin (Barc) 2004; 123:681-5.

13. Cholesterol and Statins. Bandolier 2004. Disponible en: www.jr2.ox.ac.uk/bandolier/booth/cardiac/cholstat.html (citado el13 de junio de 2005).

14. Shepherd J, Cobbe SM, Ford I, Isles CG, Lorimer AR, Macfarlane PW et al. The West of Scotland Coronary Prevention Study Group. Prevention of coronary heart disease with pravastatin in men with hypercholesterolemia. N Engl J Med. 1995; 333: 1301-8.

15. European Society of Cardiology. Heartscore, disponible en: www.escardio.org/Knowledge/decision_tools/heartscore/Program+Download.htm (citado el13 de junio de 2005)

16. Fleiss J. Statistical Methods for Rates and Proportions. 2nd edition. New York: John Willey \& Sons; 1981.p. 14-5.

17. Silva LC. Excursión a la regresión logística en ciencias de la salud. Madrid: Ediciones Díaz Santos; 1994; 97-119.

18. Feinstein AR, Cichetti DV. High agreement but low Kappa: I. The problems of two paradoxes. J Clin Epidemiol 1990; 43: 543-549.

19. Maiques Galán A. Valoración del riesgo cardiovascular. ¿Qué tabla utilizar? Aten Primaria 2003; 32 586-9.
20. Rodríguez-Artalejo F y Banegas Banegas JR. De la ecuación de Framingham a la prevención cardiovascular. Med Clin (Barc) 2003; 121: 334-6.

21. Masana Marín LL. Cálculo del riesgo cardiovascular global.¿Una utopía? Med Clin (Barc).2004; 123: $702-3$

22. Maiqués Galán A, Villar Álvarez F, Torcal Laguna J. El riesgo coronario en España y el tratamiento con fármacos hipolipemiantes. Aten Primaria 2003; 32: 420-2.

23. Mostaza JM, Vicente I, Taboada M, Laguna F, Echaniz A, García-Iglesias F et al. La aplicación de las tablas del SCORE a varones de edad avanzada triplica el número de sujetos clasificados de alto riesgo en comparación con la función de Framingham. Med Clin (Barc)2005; 124: 487-90.

24. Executive Summary of The Third report of The National Cholesterol Education Program (NCEP) Expert Panel on Detection, Evaluation, and Treatment of High Blood Cholesterol In Adults (Adult treatment Panel III). JAMA 2001; 285: 2486-97.

25. Ramos R, Marrugat J. Valoración del riesgo cardiovascular en la población. En: Del Río Ligori A, editor. Manual de Cardiología Preventiva. Madrid: SMC; 2005.

26. Aranceta J, Pérez Rodríguez C, Foz Sala M, Mantilla T, Serra Majem LL, Moreno B et al. Tablas de evaluación del riesgo coronario adaptadas a la población española. Estudio DORICA. Med Clin (Barc) 2004; 123: 686-91. 